\title{
Forensische Psychiatrie und ihre Nachbardisziplinen: Status und Zukunftsperspektiven
}

\author{
Henning Saß ${ }^{1}$
}

Online publiziert: 10. Oktober 2016

(C) Springer-Verlag Berlin Heidelberg 2016

Das nunmehr 10-jährige Erscheinen der Zeitschrift Forensische Psychiatrie, Psychologie und Kriminologie im Springer-Verlag, Heidelberg, gibt Anlass zu einer Positionsbestimmung. In der Lage der forensischen Disziplinen in Deutschland hat es im vergangenen Jahrzehnt beachtliche Fortschritte gegeben, etwa, was Forschungsergebnisse und eine Verbesserung der klinischen sowie ambulanten Versorgung angeht, aber auch beträchtliche Krisen und Gefährdungen. Beispielhaft zu nennen wären die andauernde Stigmatisierung psychisch Kranker, verstärkt durch medienträchtige Katastrophen und Straftaten, die Verunsicherungen durch Wandlungen der Diagnostik, damit verbunden wachsende Diskrepanzen zwischen medizinischen vs. rechtlichen Krankheits- oder Störungskonzepten, die Auseinandersetzungen um die Voraussetzungen und die Ausgestaltung des Maßregelvollzuges und dabei insbesondere auch der Sicherungsverwahrung sowie schließlich die Sorge um qualifizierten Nachwuchs im Versorgungs- sowie im universitären Bereich.

Angesichts dieser Situation liegt eine wichtige Zukunftsaufgabe in der Integration der verschiedenen Strömungen, Wissensbestände und Arbeitsmethoden an den Schnittstellen von Psychiatrie, Psychologie und Kriminologie mit den Rechts- sowie den Gesellschaftswissenschaften und der praktischen Justiz. Zur Diskussion dieser Fragen haben wir auf dem diesjährigen Kongress der DGPPN in Berlin zwei Jubiläumssymposien mit Vorträgen von herausragenden Vertretern unserer Fächer organisiert. Darüber hinaus

Henning $\mathrm{Sa}$

hsass@ukaachen.de

$1 \quad$ Klinik für Psychiatrie, Psychotherapie und Psychosomatik, Universitätsklinikum der RWTH, Pauwelsstr. 30, 52074 Aachen, Deutschland werden diese Themen nun in den Beiträgen dieses Heftes in erweiterter und vertiefter Form bearbeitet.

Am Anfang geht es in der Darstellung von Hans-Ludwig Kröber, Berlin, um das Verhältnis zwischen der forensischen und der allgemeinen Psychiatrie. Ganz im Sinne der Zielsetzung dieser Zeitschrift plädiert er für einen stetigen Austausch und eine enge Kooperation zwischen den Disziplinen. Die Arbeit von Dieter Seifert, Münster, und Norbert Leygraf, Duisburg/Essen, gibt einen informativen Überblick über die Entwicklung der psychiatrischen Maßregel gemäß $\S 63 \mathrm{StGB}$ in den nunmehr acht Jahrzehnten seines Bestehens. Hervorgehoben wird der Wandel von einer kustodialen Unterbringung zu einem modernen Behandlungsvollzug, der sich allerdings weiterhin einer kritischen öffentlichen Einschätzung und unterschiedlich differenzierten medialen Aktivitäten ausgesetzt sieht.

In seinem Überblick über die Situation der Kriminologie als interdisziplinäre Wissenschaft konstatiert Dieter Dölling, Heidelberg, dass diese zwar in Deutschland an den juristischen Fakultäten fest verankert ist, gleichwohl aber fallweise mit Abbautendenzen zu kämpfen hat. Gleiches gilt für andere relativ kleine Fächer im universitären Bereich, also bei uns für die Rechtspsychologie und die forensische Psychiatrie. Umso wichtiger sind die stabilen Brückenfunktionen zwischen den Rechtswissenschaften einerseits und den Psycho- und Sozialwissenschaften andererseits sowie der Anschluss an die internationalen Entwicklungen.

Klaus-Peter Dahle und Robert Lehmann, Berlin, geben einen detaillierten Überblick über die Methodenentwicklungen in den letzten Dekaden auf dem komplexen Gebiet der Kriminalprognose mit dem Wechselspiel zwischen nomothetischen und idiographischen Ansätzen. Eine wichtige Bedeutung kommt dem hier vorgestellten originär idiographischen Ansatz zu, der auf die Besonderheiten des Einzelfalles ausgerichtet ist und damit den bei uns gel- 
tenden rechtlichen Vorschriften entspricht. Aktuelle Tendenzen und künftige Entwicklungslinien der Sexualwissenschaft werden von Peer Briken, Hamburg, unter besonderer Berücksichtigung der kulturellen Dimension sexuellen Verhaltens dargestellt. Ferner behandelt er diagnostische sowie prognostische Probleme am Beispiel paraphiler Störungen und schließlich grundsätzliche Thesen zur sexualwissenschaftlichen Theoriebildung. Mit dem Spannungsfeld zwischen den Anforderungen der Besserung und der Sicherung bei den im Maßregelvollzug untergebrachten Personen befassen sich de Tribolet-Hardy und Elmar Habermeyer, Zürich. Die empirische Studienlage zur Wirksamkeit der forensisch-psychiatrischen Behandlung ergibt ein durchaus heterogenes Bild. Als wichtige Perspektive wird die wachsende Bedeutung der ambulanten Nachsorge hervorgehoben.

In der Arbeit von Kolja Schiltz et al., München/Magdeburg, geht es um die zunehmend beachtete Bedeutung neurobiologischer Untersuchungsmethoden in der forensischen Begutachtung. Wichtig erscheint die Warnung am Ende des Überblickes vor einer Überinterpretation der Befunde in Einzelfällen. Vereinfachungen und vorschnelle Verall- gemeinerungen bergen die Gefahr von Missverständnissen im forensischen Raum und können den wissenschaftlichen Kredit der Disziplin schmälern. Schließlich setzt sich Andreas Mokros, Zürich, mit der Bedeutung standardisierter Untersuchungsinstrumente in der Begutachtung auseinander und konzentriert sich dabei insbesondere auf die Beurteilung der Persönlichkeitsstruktur und die Diagnose sexueller Präferenzstörungen am Beispiel der Pädophilie. Seine entschiedenen Aussagen zur Relevanz der Testverfahren stehen in einer interessanten Spannung zu den Ausführungen von Lehmann und Dahle über die idiographischen Ansätze.

Die Lektüre der hier versammelten Arbeiten mag eine Feststellung des Editorials von Heft 1 dieser Zeitschrift im November 2006 illustrieren. Danach liegt der Reiz für Wissenschaft und Praxis in den forensischen Disziplinen in der Begegnung ganz unterschiedlicher Gebiete mit großer Eigenständigkeit in der jeweiligen Ideengeschichte, den Denkmustern, der Terminologie, den wissenschaftlichen Methoden und den Praxisfeldern. Unverzichtbar bleibt daher die Aufgabe des interdisziplinären Dialoges, des Hauptanliegens dieser Zeitschrift. 